\title{
Molecular Epidemiological Analysis of the Origin and Transmission Dynamics of the HIV-1 CRF01_AE Sub-Epidemic in Bulgaria
}

\author{
Ivailo Alexiev 1,*(D), Ellsworth M. Campbel1 ${ }^{2} \mathbb{D}$, Sergey Knyazev 2,3,4 ${ }^{\mathbb{D}}$, Yi Pan ${ }^{2}$, Lyubomira Grigorova ${ }^{1}$, \\ Reneta Dimitrova ${ }^{1}$ (D), Aleksandra Partsuneva ${ }^{1}$, Anna Gancheva ${ }^{1}$, Asya Kostadinova ${ }^{1}$, Carole Seguin-Devaux ${ }^{5}$, \\ Ivaylo Elenkov ${ }^{6}$, Nina Yancheva ${ }^{6}$ and William M. Switzer ${ }^{2}$ (D)
}

\section{check for} updates

Citation: Alexiev, I.; Campbell, E.M.; Knyazev, S.; Pan, Y.; Grigorova, L.; Dimitrova, R.; Partsuneva, A.; Gancheva, A.; Kostadinova, A.; Seguin-Devaux, C.; et al. Molecular Epidemiological Analysis of the Origin and Transmission Dynamics of the HIV-1 CRF01_AE Sub-Epidemic in Bulgaria. Viruses 2021, 13, 116. https://doi.org/10.3390/v13010116

Academic Editor: Alex Compton Received: 12 December 2020

Accepted: 14 January 2021

Published: 16 January 202

Publisher's Note: MDPI stays neutral with regard to jurisdictional claims in published maps and institutional affiliations.

Copyright: (C) 2021 by the authors. Licensee MDPI, Basel, Switzerland. This article is an open access article distributed under the terms and conditions of the Creative Commons Attribution (CC BY) license (https:// creativecommons.org/licenses/by/ $4.0 /)$
1 National Reference Laboratory of HIV, National Center of Infectious and Parasitic Diseases, 1504 Sofia, Bulgaria; lyubomiragrigorova@gmail.com (L.G.); naydenova.reneta@gmail.com (R.D.); alexandra.partsuneva@gmail.com (A.P.); gancheva.anna@gmail.com (A.G.); deshova.asi@gmail.com (A.K.)

2 Division of HIV / AIDS Prevention, National Center for HIV / AIDS, Viral Hepatitis, STD, and TB Prevention, Centers for Disease Control and Prevention, Atlanta, GA 30329, USA; ykk7@cdc.gov (E.M.C.); nvr5@cdc.gov (S.K.); jnu5@cdc.gov (Y.P.); bis3@cdc.gov (W.M.S.)

3 Department of Computer Science, Georgia State University, Atlanta, GA 30303, USA

4 Oak Ridge Institute for Science and Education, Oak Ridge, TN 37830, USA

5 Department of Infection and Immunity, Luxembourg Institute of Health, 4354 Luxembourg, Luxembourg; carole.devaux@lih.lu

6 Specialized Hospital for Active Treatment of Infectious \& Parasitic Diseases, 1606 Sofia, Bulgaria; ivayloelenkov@yahoo.com (I.E.); nyancheva@gmail.com (N.Y.)

* Correspondence: ivoalexiev@yahoo.com; Tel.: +359-2-931-8071

Abstract: HIV-1 subtype CRF01_AE is the second most predominant strain in Bulgaria, yet little is known about the molecular epidemiology of its origin and transmissibility. We used a phylodynamics approach to better understand this sub-epidemic by analyzing $270 \mathrm{HIV}-1$ polymerase (pol) sequences collected from persons diagnosed with HIV / AIDS between 1995 and 2019. Using network analyses at a $1.5 \%$ genetic distance threshold $(d)$, we found a large 154-member outbreak cluster composed mostly of persons who inject drugs (PWID) that were predominantly men. At $d=0.5 \%$, which was used to identify more recent transmission, the large cluster dissociated into three clusters of 18, 12, and 7 members, respectively, five dyads, and 107 singletons. Phylogenetic analysis of the Bulgarian sequences with publicly available global sequences showed that CRF01_AE likely originated from multiple Asian countries, with Vietnam as the likely source of the outbreak cluster between 1988 and 1990. Our findings indicate that CRF01_AE was introduced into Bulgaria multiple times since 1988, and infections then rapidly spread among PWID locally with bridging to other risk groups and countries. CRF01_AE continues to spread in Bulgaria as evidenced by the more recent large clusters identified at $d=0.5 \%$, highlighting the importance of public health prevention efforts in the PWID communities.

Keywords: HIV-1; molecular epidemiology; transmission clusters; transmission dynamics; circulating recombinant forms; drug resistance; prevention

\section{Introduction}

HIV is one of the fastest evolving RNA viruses [1]. The high HIV-1 genetic diversity is a consequence of a high replication rate, errors introduced during reverse transcription due to lack of proofreading function of the viral reverse transcriptase (RT) enzyme [2], and RT-mediated recombination between two or more distinct HIV-1 genomes in an infected person [3]. Currently, HIV-1 is divided into four phylogenetically distinct groups: $\mathrm{M}$ (major), $\mathrm{N}$ (new), $\mathrm{O}$ (outlier), and $\mathrm{P}$ representing independent zoonotic events from simian immunodeficiency virus (SIV) in chimpanzees and gorillas to HIV in humans [4]. HIV-1 
group $M$ is responsible for the current pandemic and comprises 10 distinct subtypes (A, B, C, D, F, G, H, J, K, and L), at least 102 different circulating recombinant forms (CRFs) and numerous unique recombinant forms (URFs) [5]. Globally, CRFs accounted for $16.7 \%$ of all HIV-1 infections between 2010 and 2015 and are most common in places where different subtypes co-circulate [5]. Of these CRFs, CRF01_AE has the largest global prevalence comprising $5.3 \%$ of all HIV-1 infections [5].

CRF01_AE was the first CRF identified in the HIV-1 epidemic, and it was first identified in the 1980s in sex workers in Thailand [6]. However, phylogenetic analyses demonstrated that the CRF01_AE strain first originated in Central Africa in the 1970s and then was transferred to Thailand in 1980, where it became widespread $[7,8]$. The highest prevalence of CRF01_AE (72.8\%) occurs in Southeast Asia, followed by East Asia with 47.2\% [5]. In Europe, a collaborative study among 26 European countries that included 9588 samples from patients diagnosed between 2008 and 2010 showed a 3.2\% prevalence of CRF01_AE [9]. Further studies conducted in Bulgaria have found a relatively high prevalence of CRF01_AE, indicating that Bulgaria probably has the highest prevalence of this strain in Europe with around 20\% CRF01_AE infections [10-12]. Moreover, the dissemination of CRF01_AE was not evenly distributed in different vulnerable groups and regions of the country but rather prevailed among PWID in the Sofia region by 35\% of HIV-1 infections [13].

While our initial study importantly identified a subpopulation infected with CRF01_AE in Bulgaria, the analysis was limited to 41 infected persons with samples collected between 1999 and 2011 [11]. Since then, the number of CRF01_AE infections has increased 6.6-fold, representing a significant public health burden and necessitating further understanding of the underlying transmission dynamics of this subtype in Bulgaria. The aim of our current study was to characterize the transmission dynamics of the CRF01_AE sub-epidemic in Bulgaria by combining and analyzing available HIV-1 epidemiological and nucleotide sequence data to infer its origin and transmission events and to help inform public health prevention strategies.

\section{Materials and Methods}

\subsection{Study Design and Patient Samples}

Following national standards consistent with guidelines of European AIDS Clinical Society, the genotypic resistance testing of HIV-1 patients in Bulgaria was performed as soon as possible after diagnosis or as a clinical follow-up after virological failure. The tests were conducted randomly, regardless of sex, age, or transmission group of the patients. Fresh, whole blood samples from individuals with HIV-1 diagnosed between 1995 and 2019 were collected during the diagnostic process and clinical follow-up at the National Reference Confirmatory Laboratory of HIV (NRCL of HIV) Sofia, Bulgaria. Demographic and epidemiological information were collected using patient self-assessment interviews following national regulations. Blood samples were linked to demographic and clinical data by using anonymous numerical codes in accordance with the ethical standards of Bulgaria as previously described [11].

\subsection{Dataset and Sequence Analyses}

Blood samples were processed for plasma aliquots that were kept frozen at $-80{ }^{\circ} \mathrm{C}$ until used. Viral RNA was isolated from plasma samples using the Abbott ViroSeq HIV1 Genotyping Test and/or QIAmp Viral RNA Mini Kit. The HIV-1 protease and part of reverse transcriptase regions of the pol gene was sequenced using the ViroSeq HIV-1 Genotyping Test (Abbott) using the Applied Biosystems 3130xl genetic analyzer or TruGene DNA Sequencing System (Siemens Healthcare Medical Solutions Diagnostics, Germany) and an OpenGene DNA sequencing system following the manufacturer's protocol [13].

HIV-1 CRF01_AE subtype was defined using a set of methods, including the automated subtype tool COMET v2.2 [14], the REGA HIV-1 subtyping tool version 3.0 [15], the jumping profile Hidden Markov Model (jpHMM) [16], and SimPlot [17]. 
Sequence alignments were performed using the MUSCLE algorithm implemented in AliView version $1.23[18,19]$ and MAFFT version $7[20,21]$. Additional editing of the alignments was performed manually. The complete dataset contained 270 Bulgarian CRF01_AE HIV-1 sequences. All Bulgarian HIV-1 CRF01_AE sequences were deposited in GenBank with the following accession numbers: EF517429-EF517431, EF517441, EF517442, EF517444, EF517448, EF517454, EF517455, EF517461, EF517463, EF517469, EF517471, EF517476, EF517477, EF517479, EF517480, EF517486, JQ259065, JQ259069, JQ259071, JQ259083, JQ259084, JQ259089, JQ259094, JQ259107, JQ259115-JQ259117, JQ259121, JQ259128, JQ259148, JQ259167, JQ259171JQ259173, JQ259177, KJ765390, KJ765392, KJ765395, KJ765398, KJ765403, KJ765404, KJ765414, KJ765416, KJ765422, KJ765423, KJ765425, KJ765429, KJ765431, KJ765433, KJ765437, KJ765439, KJ765445, KJ765447, KJ765463, KJ765474, KJ765483, KJ765486, KJ765487, KJ765488, KJ765490, KJ765493, KJ765497, KJ765498, KJ765500, KJ765505, KJ765510, KJ765514, KJ765515, KJ765538, KJ765549, KJ765552, KJ765564, KJ765572, KJ765579-KJ765581, KJ765583, KJ765588, KJ765594, KJ765598, KJ765600, KJ765602, KJ765606, KT805898, KT805901, KT805902, KT805904, and MW196446-MW196626.

The potential origin of the HIV-1 CRF01_AE clades in Bulgaria was evaluated by phylogenetic analyses. Approximate maximum likelihood (ML) phylogenies were reconstructed using all 270 Bulgarian pol isolates, and 115 sequences from a BLAST search at GenBank to the Bulgarian sequences (top 10 hits for each sequence with $>95 \%$ sequence identity and de-duplicated) and $1343 \mathrm{HIV}-1$ pol sequences from the Los Alamos database from 2019 excluding any duplicates, using the GTR nucleotide substitution model in FastTree v2.1.10 [22]. We used a local instance of Nextstrain to conduct viral dating and evolutionary rate analyses. Nextstrain uses ML analysis implemented in TreeTime $[23,24]$ and is used for monitoring emerging outbreaks such as COVID-19 in near-real time (https://nextstrain.org/). Three HIV-1 subtype J sequences were used as the outgroup for the Nextstrain ML analysis. The total dataset for phylogenetic analysis contained 1731 HIV-1 sequences.

Identification of CRF01_AE clusters and characterization of the transmission network was done using the sequence alignment and MicrobeTrace (http:/ / github.com/cdcgov / microbetrace) [25] at Tamura-Nei genetic distance (d) cutoffs of 0.005 (0.5\%), 0.015 (1.5\%), and $0.035(3.5 \%)$ nucleotide/substitutions/site. Graphically, transmission linkages are represented by lines drawn between both nodes, where each node represents a participant's pol sequence. If a participant's pol sequence was linked to another according to a specific threshold, both participants were labeled as clustered. Those participants whose pol sequence did not link to any other participant were labeled unclustered or singletons. Categorical and numeric assortativity coefficients for selected variables among the Bulgarian cases were calculated using the Python package https:/ / github.com/Sergey-Knyazev/ attribute_assortativity [26] NetworkX (https:/ / networkx.github.io/) [27] and thresholds of $\mathrm{d}=0.5 \%, 1.0 \%, 1.5 \%$, and $2.0 \%$.

\subsection{Statistical Analysis}

Epidemiological characteristics such as sex, age, country of birth, likely country of infection, region in Bulgaria, and transmission categories were considered. The frequencies as well as percentages were analyzed by infection with CRF01_AE and other HIV-1 subtypes. The association between CRF01_AE infection and the characteristics were evaluated by Fisher's exact test, as sample sizes were small. Multivariable analysis was performed with logistic regression with the outcome variable as subtype CRF01_AE or other subtypes. Country of origin was excluded due to low numbers of infections outside of Bulgaria. Age at diagnosis was treated as continuous variable in the multivariable analysis. Odds ratios were estimated with $95 \%$ confidence intervals (CIs). All analyses were performed in SAS v9.4 (SAS Institute, Cary, NC, USA). 


\section{Results}

\subsection{Characteristics of the HIV-1 CRF01_AE Infections in Bulgaria}

The first HIV-1 CRF01_AE infection in Bulgaria was diagnosed in 1995 in a male reporting only heterosexual behaviors, and for the next 11 years, only 33 cases with this subtype were identified, most (81.8\%) of whom were heterosexual (HET). In 1999, CRF01_AE was first found in a mother-to-child transmission (MTC), and in 2002, it had spread to PWID. In 2009, 26 individuals with CRF01_AE infection were identified, 18 $(69.2 \%)$ of which were PWID and $3(11.5 \%)$ were men who have sex with men and inject drugs (MSM/PWID), leading to an outbreak among this vulnerable population in the region of the capital Sofia (Figure 1). The number of CRF01_AE infections continued to increase through 2011 with 31 cases identified that year, including 17 PWID, 12 HET, and two MSM. Following this outbreak, emergency measures were taken by the government to limit the spread of HIV in this group, and in the following years, the number of registered PWIDs and individuals from other transmission groups infected with CRF01_AE decreased. Nonetheless, starting in 2018, the number of cases began to increase again with 17 (9 PWID) in 2018 and 22 (14 PWID) in 2019.

\section{Epidemiologic Curve by Transmission Category}

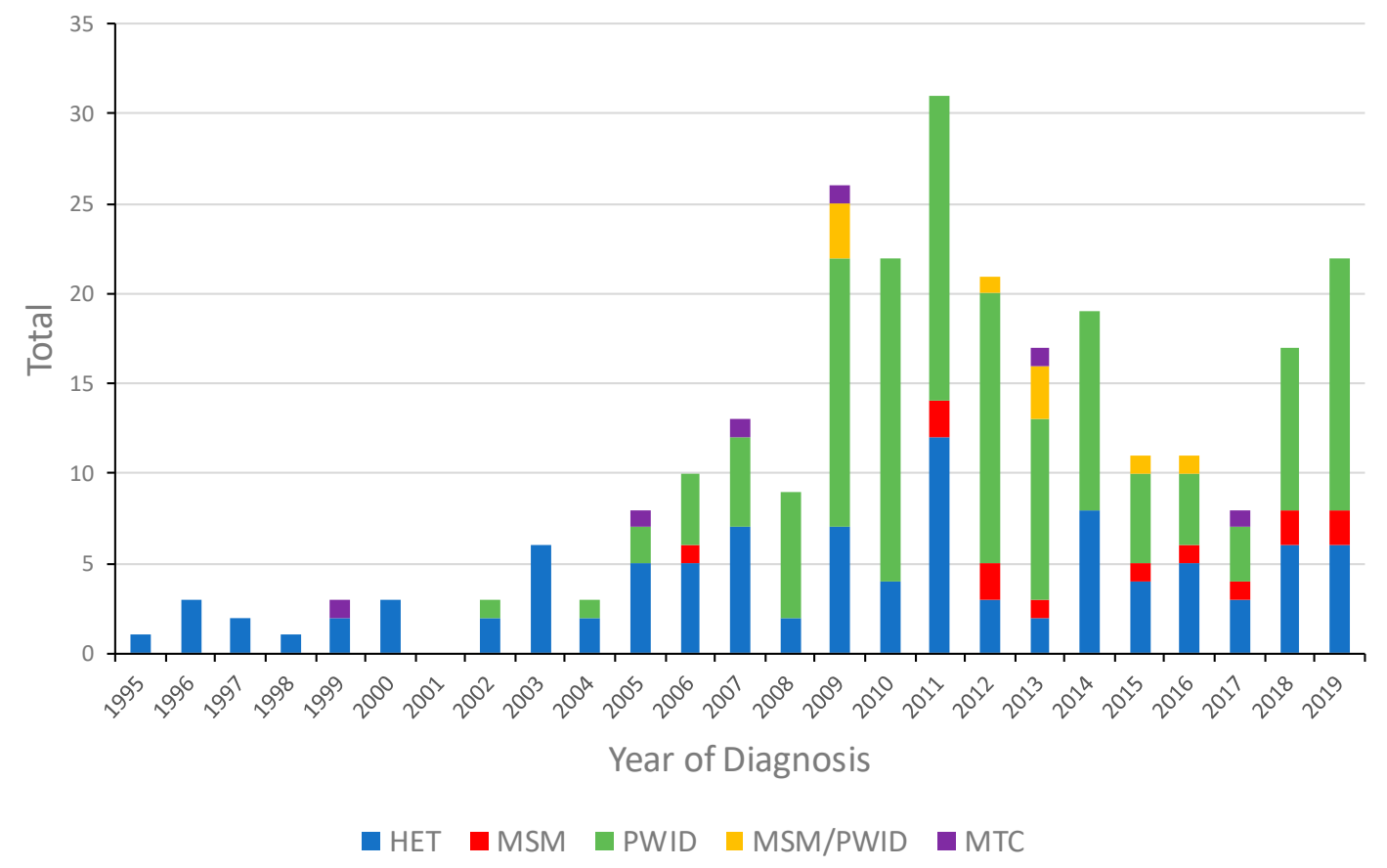

Figure 1. Noncumulative epidemiologic curve of HIV-1 CRF01_AE diagnoses in Bulgaria from 1995 to 2019 by transmission category. HET, heterosexual; MSM, men who have sex with men; PWID, persons who inject drugs; MSM/PWID (persons who reported both MSM and PWID); MTC, mother-to-child.

By the end of 2019, CRF01_AE infection was found in 270 individuals, 187 (69.3\%) of whom were male and $83(30.7 \%)$ of whom were female (Table 1$)$. The proportion of women in the group of people infected with CRF01_AE (30.7\%) was statistically higher compared to individuals infected with another HIV subtype (14.4\%). Age at diagnosis for persons with CRF01_AE infection ranged from 0 to $63($ mean $=30.0$, median $=29.0)$ with the proportion of young participants (up to 19 years) twice as high (10.4\%) than those infected with other subtypes (4.8\%). Based on patient interviews, $251(93 \%)$ of the infections were presumed to have occurred in Bulgaria, whereas only $19(7 \%)$ occurred in other countries, indicating that more CRF01_AE infections occurred locally (93\%) than the acquisition of HIV from another subtype $(83.2 \%, p<0.0001)$. With one exception of 
infection likely occurring in Thailand, all other cases of transmission outside the country are from Europe, including France $(n=4)$, Germany $(n=3)$, Greece $(n=2)$, Spain $(n=4)$, Serbia $(n=1)$, Russia $(n=1)$, Turkey $(n=1)$, and the United Kingdom $(U K ; n=2)$. We found that participants with CRF01_AE infection had significantly lower percentages of MSM (4.8\% vs. $44.6 \%$ ) and higher percentages of PWID (52.2\% vs. $11.2 \%)$ compared to non-CRF01_AE participants (Table 1). The percentage of HET infections with CRF01_AE was like that from other subtypes ( $37.4 \%$ vs. $41.9 \%)$.

Table 1. Epidemiological characteristics of individuals infected with HIV-1 CRF01_AE compared to infection with other HIV-1 subtypes and circulating recombinant forms (CRFs) in Bulgaria.

\begin{tabular}{|c|c|c|c|}
\hline Characteristic & $\begin{array}{c}\text { Subtype CRF01_AE } \\
\text { n (\%) }\end{array}$ & $\begin{array}{c}\text { Other Subtypes } \\
\text { n (\%) }\end{array}$ & $p$ Value \\
\hline Total & 270 & 1413 & \\
\hline Sex & & & $<0.0001$ \\
\hline Men & 187 (69.3) & 1195 (84.6) & \\
\hline Women & $83(30.7)$ & $218(15.4)$ & \\
\hline Age (years) & & & 0.001 \\
\hline$\leq 19$ & $28(10.4)$ & $68(4.8)$ & \\
\hline $20-29$ & $108(40.0)$ & $538(38.1)$ & \\
\hline $30-39$ & $90(33.3)$ & 493 (34.9) & \\
\hline $40-49$ & $35(13.0)$ & $204(14.4)$ & \\
\hline$\geq 50$ & $9(3.3)$ & $110(7.8)$ & \\
\hline Country of Birth & & & 0.0018 \\
\hline Bulgaria & $269(99.6)$ & $1361(96.3)$ & \\
\hline Other country & $1(0.4)$ & $52(3.7)$ & \\
\hline $\begin{array}{l}\text { Likely Country of } \\
\text { Infection }\end{array}$ & & & $<0.0001$ \\
\hline Bulgaria & $251(93.0)$ & $1176(83.2)$ & \\
\hline Other country $^{2}$ & $19(7.0)$ & $237(16.8)$ & \\
\hline Region in Bulgaria & & & $<0.0001$ \\
\hline Sofia & $164(60.7)$ & $625(44.2)$ & \\
\hline Other regions & $106(39.3)$ & $788(55.8)$ & \\
\hline $\begin{array}{c}\text { Transmission } \\
\text { category }^{3}\end{array}$ & & & $<0.0001$ \\
\hline HET & $101(37.4)$ & $592(41.9)$ & \\
\hline MSM & $13(4.8)$ & $630(44.6)$ & \\
\hline PWID & $141(52.2)$ & $158(11.2)$ & \\
\hline Other & $15(5.6)$ & $33(2.3)$ & \\
\hline
\end{tabular}

Percentages are provided in parentheses. ${ }^{2}$ Other countries include France $(n=4)$, Germany $(n=3)$, Greece $(n=$ 2), Spain ( $(n=4)$, Serbia $(n=1)$, Russia $(n=1)$, Thailand $(n=1)$, Turkey $(n=1)$, and the United Kingdom $(n=2) .{ }^{3}$ HET, heterosexual; MSM, men who have sex with men; PWID, persons who inject drugs; Other includes MSM + PWID (persons who reported both MSM and PWID); mother-to-child (MTC), and blood transfusion (BLD). Fisher's exact test was applied to calculate $p$ values.

Our multivariable analysis of CRF01_AE infection in Bulgaria compared to other subtypes confirmed these findings (Table 2). HIV-1-infected persons in Bulgaria were more likely to have subtype CRF01_AE compared to those residents infected in another country $(\mathrm{OR}=1.90,95 \% \mathrm{CI}=(1.11,3.27), p=0.02)$. Similarly, patients in Sofia were more likely to 
have CRF01_AE infection compared to persons residing in other regions of Bulgaria (OR = $2.98,95 \% \mathrm{CI}=(2.19,4.05), p=0.02)$. Furthermore, the odds of having CRF01_AE infection in PWID compared to HET was $6.40(95 \% \mathrm{CI}=(4.41,9.28), p<0.0001)$ and the odds of having CRF01_AE infection in persons reporting both MSM and PWID risks compared to HET was $3.44(95 \% \mathrm{CI}=(1.64,7.23), p<0.0001)$. We did not observe an association of age at diagnosis with subtype infection.

Table 2. Multivariable analysis of selected demographic characteristics comparing HIV-1 CRF01_AE infection to that with other subtypes in Bulgaria.

\begin{tabular}{|c|c|c|c|c|}
\hline Characteristic & Odds Ratio & \multicolumn{2}{|c|}{$95 \%$ CI } & $p$ Value \\
\hline Sex (Women vs. Men) & 2.14 & 1.48 & 3.08 & $<0.0001$ \\
\hline Age at diagnosis (in years) & 1.00 & 0.99 & 1.02 & 0.73 \\
\hline $\begin{array}{l}\text { Likely country of infection } \\
\text { (Bulgaria vs. Other) }\end{array}$ & 1.90 & 1.11 & 3.27 & 0.02 \\
\hline Region in Bulgaria (Sofia vs. Other) & 2.98 & 2.19 & 4.05 & $<0.0001$ \\
\hline Transmission category & & & & $<0.0001$ \\
\hline$M S M$ vs. HET & 0.13 & 0.07 & 0.24 & $<0.0001$ \\
\hline PWID vs. HET & 6.40 & 4.41 & 9.28 & $<0.0001$ \\
\hline Other vs. HET & 3.44 & 1.64 & 7.23 & 0.001 \\
\hline
\end{tabular}

CI, confidence interval. Country of origin was excluded from the analysis due to the sparseness of infections outside of Bulgaria. Age at diagnosis was treated as continuous variable in the multivariable analysis. HET, heterosexual; MSM, men who have sex with men; PWID, persons who inject drugs; Other includes MSM + PWID (persons who reported both MSM and PWID).

\subsection{Identification and Characterization of HIV-1 Subtype CRF01_AE Transmission Clusters in Bulgaria}

We used MicrobeTrace to infer HIV transmission clusters at two genetic distance thresholds to capture genetically related established and recent infections $(1.5 \%)$ and then to identify more recent transmission linkage (0.5\%) within Bulgaria (Figure 2). At the $1.5 \%$ cutoff, six clusters were inferred, including one large cluster containing 154 members (Table 3, Figure 2A). This large cluster consisted of mostly men (116/154, 75.3\%) and PWID $(108 / 154,70.1 \%)$ and contained CRF01_AE-infected persons with the lowest mean and median ages at diagnosis. These results contrast with those from CRF01_AE pol sequences that did not cluster at this threshold, which were more evenly distributed between the sexes (57 males, 41 females), and dominated by HET (64/98, 65.3\%) (Table 3). The large cluster also had slightly more individuals reporting HIV-1 infection in other countries $(11 / 154,7.1 \%)$ compared to $(6 / 98,6.1 \%)$ for pol sequences that did not cluster (Table 3$)$. The large cluster also contained persons with HIV diagnoses between 2002 and 2019 (Table 3). 


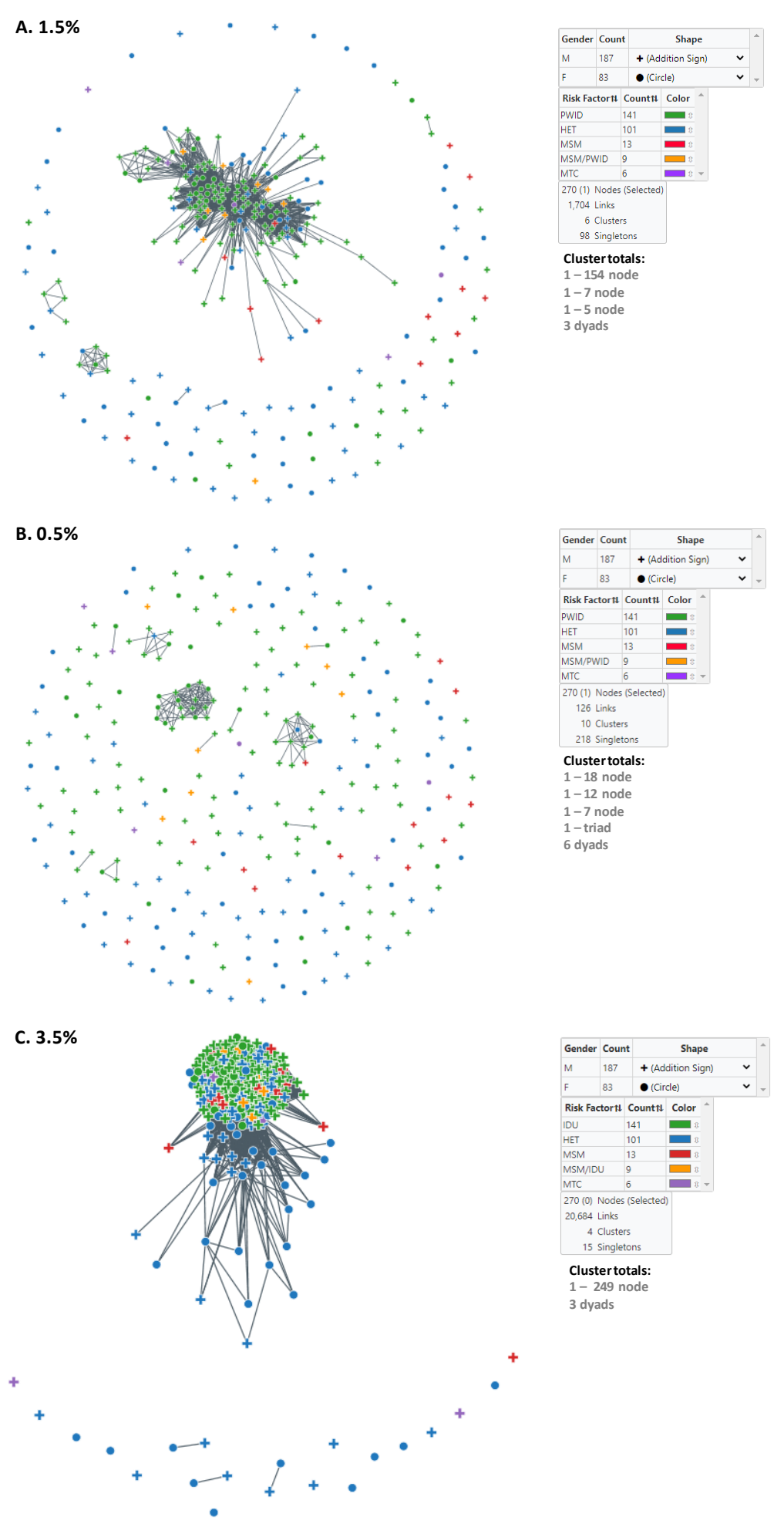

Figure 2. Inference of subtype CRF01-AE clusters in Bulgaria using MicrobeTrace. (A) Six clusters were identified using a genetic distance $(d)$ of $1.5 \%$ compared to $(\mathbf{B})$ ten clusters at $d=0.5 \%$. Sex is indicated by circles (male) and addition sign (female). (C) Transmission network at $d=3.5 \%$. Transmission category is indicated with color (red, men who have sex with men (MSM); green, persons who inject drugs (PWID); blue, heterosexual (HET); gold, persons reporting both MSM and PWID; purple, mother-to-child (MTC). Cluster totals by node (members) and total number of links in the transmission network is provided. 
Table 3. Characteristics of HIV-1 subtype CRF01_AE clusters and unclustered persons in Bulgaria, 1995-2019, using genetic distance cutoffs of $1.5 \%$, $0.5 \%$, and 3.5\% ${ }^{1}$.

\begin{tabular}{|c|c|c|c|c|c|c|c|c|c|c|c|c|}
\hline & $\begin{array}{c}\text { Cluster Sizes at } \\
1.5 \%\end{array}$ & Male & Female & HET & MSM & MSM/PWID & PWID & Vertical & $\begin{array}{l}\text { Mean/Median } \\
\text { Age at Diagnosis }\end{array}$ & $\begin{array}{c}\text { Diagnosis } \\
\text { Date Range }\end{array}$ & $\begin{array}{l}\text { Likely Country of } \\
\text { Infection (Bulgaria) }\end{array}$ & $\begin{array}{l}\text { Likely Country of } \\
\text { Infection (Other) }\end{array}$ \\
\hline & 154 & 116 & 38 & 30 & 5 & 8 & 108 & 3 & $28.4 / 29.0$ & 2002-2019 & 143 & 11 \\
\hline & 7 & 5 & 2 & 2 & 0 & 0 & 5 & 0 & $38.9 / 39.0$ & 2018-2019 & 6 & 1 \\
\hline & 5 & 5 & 0 & 1 & 0 & 0 & 4 & 0 & $33.0 / 32.0$ & 2010-2019 & 5 & 0 \\
\hline & Three dyads (6 total) & 4 & 2 & 4 & 0 & 0 & 2 & 0 & $38.8 / 32.5$ & 1999-2019 & 5 & 1 \\
\hline & Singletons (98 total) & 57 & 41 & 64 & 8 & 1 & 22 & 3 & $31.2 / 31.0$ & 1995-2019 & 92 & 6 \\
\hline \multirow{6}{*}{ Totals } & $\begin{array}{c}\text { Cluster Sizes at } \\
0.5 \%\end{array}$ & Male & Female & HET & MSM & MSM/PWID & PWID & Vertical & $\begin{array}{c}\text { Mean/Median } \\
\text { Age at Diagnosis }\end{array}$ & $\begin{array}{c}\text { Diagnosis } \\
\text { Date Range }\end{array}$ & $\begin{array}{l}\text { Likely Country of } \\
\text { Infection (Bulgaria) }\end{array}$ & $\begin{array}{l}\text { Likely Country of } \\
\text { Infection (Other) }\end{array}$ \\
\hline & 18 & 10 & 8 & 0 & 0 & 0 & 18 & 0 & $22.5 / 20.5$ & 2009-2018 & 17 & 1 \\
\hline & 12 & 9 & 3 & 2 & 1 & 0 & 9 & 0 & $30.3 / 31.0$ & 2009-2015 & 10 & 2 \\
\hline & 7 & 6 & 1 & 1 & 0 & 0 & 6 & 0 & $27.9 / 29.0$ & 2011-2015 & 7 & 0 \\
\hline & 3 & 2 & 1 & 0 & 0 & 0 & 3 & 0 & $38.0 / 38.0$ & $2018-2019$ & 2 & 1 \\
\hline & Singletons (218 total) & 151 & 67 & 98 & 12 & 7 & 96 & 5 & $30.7 / 30.0$ & 1995-2019 & 203 & 15 \\
\hline \multirow[t]{5}{*}{ Totals } & 270 & 187 & 83 & 101 & 13 & 9 & 141 & 6 & $30.0 / 29.0$ & 1995-2019 & 251 & 19 \\
\hline & $\begin{array}{c}\text { Cluster Sizes at } \\
3.5 \%\end{array}$ & Male & Female & HET & MSM & MSM/PWID & PWID & Vertical & $\begin{array}{c}\text { Mean/Median } \\
\text { Age at Diagnosis }\end{array}$ & $\begin{array}{c}\text { Diagnosis } \\
\text { Date Range }\end{array}$ & $\begin{array}{l}\text { Likely Country of } \\
\text { Infection (Bulgaria) }\end{array}$ & $\begin{array}{l}\text { Likely Country of } \\
\text { Infection (Other) }\end{array}$ \\
\hline & 249 & 176 & 73 & 83 & 12 & 9 & 141 & 4 & $30.0 / 29.0$ & 1995-2019 & 234 & 15 \\
\hline & Three dyads (6 total) & 3 & 3 & 6 & 0 & 0 & 0 & 0 & $30.3 / 30.0$ & 2006-2019 & 4 & 2 \\
\hline & Singletons (15 total) & 8 & 7 & 12 & 1 & 0 & 0 & 2 & $29.5 / 29.0$ & 1998-2018 & 13 & 2 \\
\hline Totals & 270 & 187 & 83 & 101 & 13 & 9 & 141 & 6 & $30.0 / 29.0$ & 1995-2019 & 251 & 19 \\
\hline
\end{tabular}

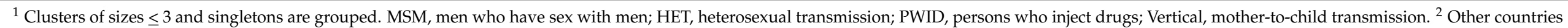
include France $(n=4)$, Germany $(n=3)$, Greece $(n=2)$, Spain $(n=4)$, Serbia $(n=1)$, Russia $(n=1)$, Thailand $(n=1)$, Turkey $(n=1)$, and the United Kingdom $(n=2)$. 
At the more stringent $0.5 \%$ cutoff, an additional four clusters were identified as a result of all six clusters at the $1.5 \%$ genetic distance disassociating at the lower threshold into smaller clusters or completely into singletons (Table 3, Figure 2B). The large 154-member cluster at the $1.5 \%$ genetic distance cutoff was reduced to the 18,12 , and 7 member clusters, five dyads, and 107 singletons at the $0.5 \%$ threshold. The seven-member cluster at the $1.5 \%$ cutoff with the most recent HIV diagnoses did not completely dissociate but rather was reduced to one triad, one dyad, and two singletons (Table 3). The five-member cluster and the three dyads completely dissociated. At the $0.5 \%$ cutoff, all clusters contained mostly PWID and consisted of persons diagnosed from 2009-2019 (Table 3).

Notably, cluster analysis at the $1.5 \%$ distance threshold did not capture all the Bulgarian sequences found in the large outbreak cluster identified using phylogenetic analysis described below. Once we increased the cutoff to $d=3.5 \%$, most of the phylogenetic outbreak cluster sequences were in a single, large network cluster of 249 nodes (Figure 2C). All PWID were in the 249-member cluster as were most infections reportedly acquired abroad and included infections across all years of diagnosis. At this threshold, there were also three dyads and 15 singletons that were composed of mostly HETs (Table 3). The dyads were diagnosed with HIV infection between 2006 and 2019.

\subsection{Assortative Mixing of Pairs, Sex, Similar Ages, Transmission Category, and Geographic Location in the HIV-1 CRF01_AE Transmission Networks}

Assortativity is a quantitative measure often used to help characterize cluster composition that describes the likelihood that a node in a network is connected to a node bearing similar characteristics. Assortativity coefficient (r) values of 1.0 indicate perfect assortativity, while at $r=-1.0$, the network is completely disassortative, and when $r=0$, the network is non-assortative. Our analysis found that clusters have high assortativity by region $(\mathrm{r}>0.25)$, at both strict $(d=0.005)$ and relaxed $(d=0.015)$ thresholds, in most size categories and across the network in aggregate (Table 4). Region assortativity is higher for $d=0.005$ than for $d=0.015$ for most clusters, showing the prevalence of local transmission linkage in the network. However, region was not assortative for dyads at a strict threshold and for clusters of size 3-9 at a relaxed threshold. Sex was strongly disassortative for dyads under both threshold measures $(<-0.30)$ and was disassortative for clusters size 3-9 at the strict threshold. Sex was not disassortative for larger clusters or the full data set. The transmission category displayed a mix of both assortativity and disassortativity, depending on the threshold and cluster size category. Dyads at strict threshold indicate a mix of transmission categories between pairs, whereas dyads at a relaxed threshold showed concurrence among transmission category between pairs. Among clusters of size 3-9, transmission category was disassortative, regardless of threshold. 
Table 4. Assortative mixing of HIV-1 subtype CRF01_AE clusters in Bulgaria by region, sex, and transmission category ${ }^{1}$.

\begin{tabular}{|c|c|c|c|c|c|c|c|c|c|c|c|c|c|c|c|c|c|c|c|c|c|c|c|c|}
\hline & \multicolumn{24}{|c|}{ Cluster Size, Genetic Distance $(d)$ Threshold, Total Persons } \\
\hline & Size & $d^{2}$ & Total $^{3}$ & Size & $d$ & Total & Size & $d$ & Total & Size & $d$ & Total & Size & $d$ & Tota & Size & $d$ & Total & Size & $d$ & Total & Size & $d$ & Total \\
\hline $\begin{array}{c}\text { Cluster } \\
\text { Characteristic }\end{array}$ & dyad & 0.5 & 12 & dyad & 1.5 & 6 & $\begin{array}{l}3- \\
9\end{array}$ & 0.5 & 10 & $3-9$ & 1.5 & 12 & $\geq 10$ & 0.5 & 30 & $\geq 10$ & 1.5 & 154 & All & 0.5 & 52 & All & 1.5 & 172 \\
\hline Region & & 0.05 & & & 1 & & & 0.41 & & & -0.07 & & & 0.72 & & & 0.29 & & & 0.66 & & & 0.28 & \\
\hline Sex & & -0.33 & & & -0.5 & & & -0.24 & & & -0.07 & & & -0.03 & & & 0.01 & & & -0.05 & & & 0 & \\
\hline $\begin{array}{c}\text { Transmission } \\
\text { category }\end{array}$ & & -0.24 & & & 1 & & & -0.3 & & & -0.2 & & & -0.04 & & & 0.03 & & & -0.06 & & & 0.03 & \\
\hline
\end{tabular}

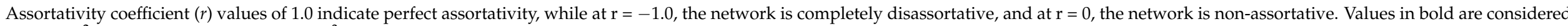
significant. ${ }^{2} d$, genetic distance percentage. ${ }^{3}$ Total number of nodes or sequences. 


\subsection{Origin of HIV-1 CRF01_AE Infections in Bulgaria}

A total of 1458 global CRF01_AE HIV-1 pol sequences were used to infer the phylogenetic relationships of the 270 Bulgarian CRF01_AE sequences from our study with those from other countries to evaluate potential origins of the subtype CRF01_AE sub-epidemic in Bulgaria (Figure 3). The phylogenetic tree generated by ML analysis in Nextstrain overall shows that the majority of CRF01_AE transmission events are taking place in Asia with some regional isolation after local introduction, as seen for China, but with spread and mixing in other geographic locations as for infections in Thailand and Vietnam (Figure 3A). For Bulgaria, isolated cases of CRF01_AE infection $(n=19)$ with HIV diagnoses between 1995 and 2019 showed phylogenetic linkage to sequences from Thailand, Vietnam, and China, and for one case, Sweden. Of these 19 cases, 16 (84.2\%) reported only HET as the transmission risk factor, of which two reported acquiring infection outside Bulgaria. Two others were children infected by mother-to-child transmission, and one was an MSM.

Nextstrain ML analysis identified a large clade containing the majority of the Bulgarian CRF01_AE sequences (Figure 3A). This clade consisted of two subclades with a larger subclade clade composed of 248 Bulgarian sequences from persons diagnosed between 1996 and 2019 and 11 non-Bulgarian sequences. We refer to this larger subclade as the CRF01_AE outbreak cluster. Near the root of the outbreak cluster are four CRF01_AE sequences from China from 2005 to 2007, and three from Vietnam from 2012. Within the outbreak clade are two sequences, which were each from the Czech Republic from 2005 and the UK from 2013. The only epidemiologic data available were three of the sequences from China, which included two females and one male, all HET in their twenties [28]. The smaller subclade contained four Bulgarian sequences from persons diagnosed between 2011 and 2016 and 15 non-Bulgarian sequences.

The outbreak cluster also included two Bulgarian sequences from a man and woman with no risk behaviors identified from a previous study by a different group [29]. Most cases $(64.1 \%, 159 / 248)$ in the outbreak clade were from the capital city of Sofia of which $108 / 159$ (67.9\%) were PWID. PWID predominated the risk factor in the outbreak clade $(60.5 \%, 150 / 248)$, which was followed by HET $(34.3 \%, 85 / 248), \operatorname{MSM}(4.8 \%, 12 / 248)$, and MTC $(1.6 \%, 4 / 248)$.

Molecular dating inferred the most recent common ancestor (MRCA) for the outbreak clade to $12 / 21 / 88$ with a confidence interval (CI) between $4 / 20 / 88$ and $3 / 27 / 90$. The MRCA for the small clade was estimated to have occurred on 3/11/89 (CI 6/4/88-6/10/90). The MRCA for the complete clade of 278 taxa was $8 / 31 / 88$ (CI 12/2/87-11/25/89) compared to 1/18/74 (CI 9/152/71-12/22/78) for the entire CRF01_AE clade. The evolutionary rate for our CRF01_AE dataset was estimated to be 1.23E-03 nucleotide substitutions/site/year.

Figure 3B shows the inferred transmission routes for the origin and spread of the outbreak cluster using Nextstrain. The turquoise line from Vietnam to Bulgaria indicates that the original HIV-1 source of the outbreak cluster sequences in Bulgaria likely originated from Vietnam. As the outbreak in PWIDs grew in Bulgaria, HIV transmission from Bulgaria spread back to Vietnam, and to China, the UK, and the Czech Republic as indicated by the red lines from Bulgaria to these four countries. 

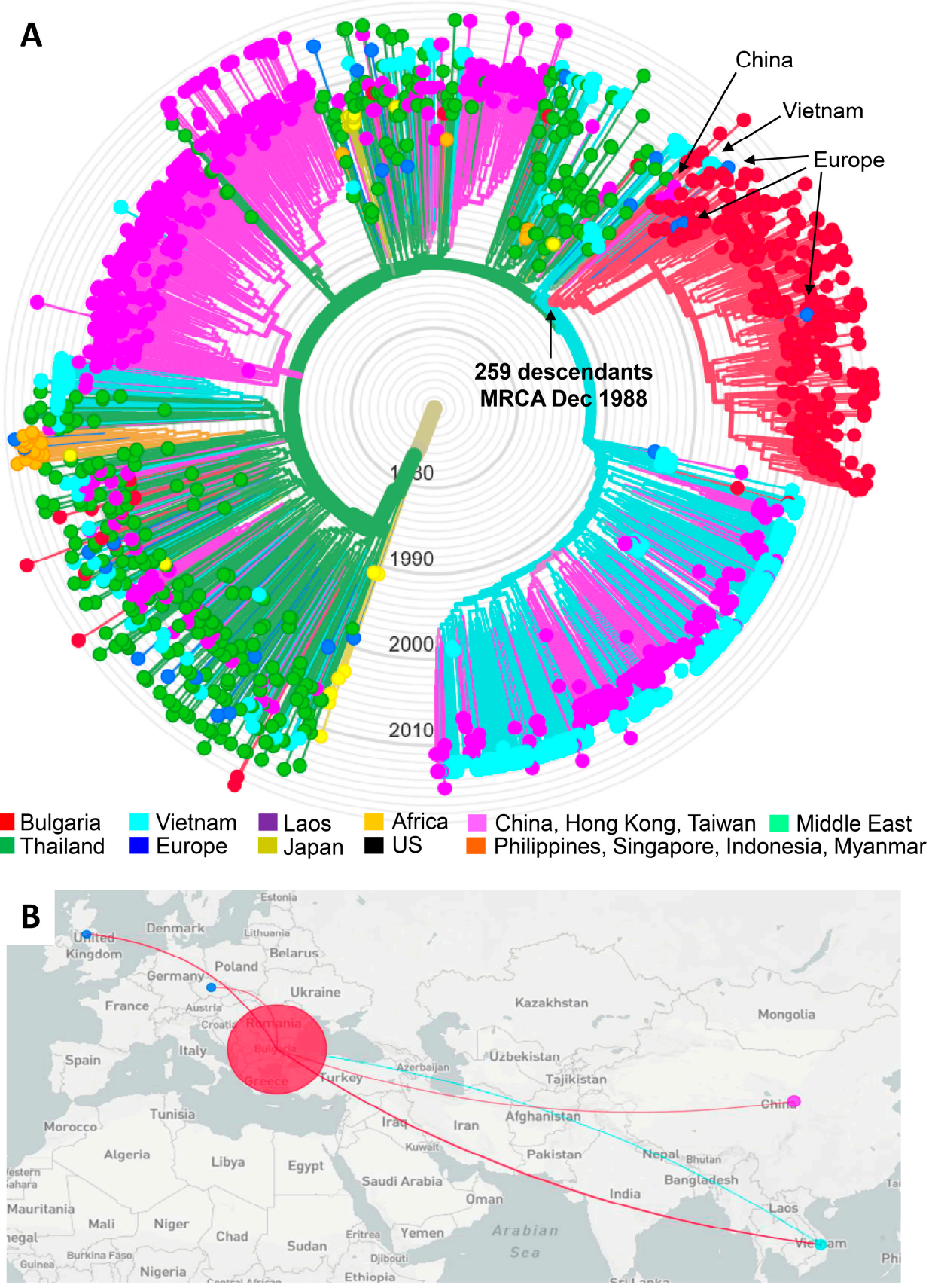

Figure 3. Nextstrain maximum-likelihood (ML) analysis of global HIV-1 CRF01_AE sequences. (A) The ML tree was constructed using 270 sequences from Bulgaria and 1458 global CRF01_AE sequences, including two from Bulgaria from a different study, 7 from Africa (Cameroon $=5$, the Central African Republic =2), 544 from China, 1 from Hong Kong, 3 from Taiwan, 20 from Europe (Belgium = 2, Czech Republic $=2$, Finland $=1$, France $=1$, Slovenia $=1$, Sweden $=7$, the United Kingdom = 6), 276 from Vietnam, 49 from Laos, 362 from Thailand, 19 from the Philippines, 2 from Singapore, 3 from Indonesia, 2 from Myanmar, 6 from the United States, and two from the Middle East (Afghanistan = 1, Iran = 1). Three reference subtype J sequences were used as the outgroup. Tree branches are colored by country of birth as provided in the key. The arrows show the Bulgarian outbreak cluster showing inferred dating of the most recent common ancestor (CI, confidence interval). (B) Inferred geographical transmission routes with potential origins indicated by colored lines connecting countries, as provided in the (A). 


\section{Discussion}

In this study, we reconstructed and analyzed transmission networks of CRF01_AE, the most prevalent recombinant form of HIV-1 in Bulgaria, by combining traditional epidemiological data with HIV-1 pol sequences collected through 2019 and conducting phylogenetic and network analyses. Our phylodynamic approach has been used successfully before for the subtype B sub-epidemic in Bulgarian [30] and these methods are becoming standard analyses for cluster and outbreak investigations for HIV prevention [31-34]. We found that CRF01_AE infections grew into a large sub-epidemic among PWID, after it was first introduced into Bulgaria, most likely from Vietnam, with some onward transmission to other European countries.

The first CRF01_AE infection in Bulgaria was diagnosed in 1995 mostly in HET individuals $(81.8 \%)$, keeping the spread of this subtype to a limited extent. However, in 2002, CRF01_AE was introduced into PWID and then spread rapidly among this vulnerable population, leading to local outbreak in the capital Sofia in 2009 (Figure 1) [11-13]. Since then, CRF01_AE has become the second most predominant subtype in Bulgaria, which to our knowledge represents the highest share of CRF01_AE in Europe [5,9]. Our results resemble those described for other HIV-1 outbreaks in PWID in multiple countries, including Greece, Luxembourg, and resulting from the opioid crisis in the United States with the introduction of specific subtypes into this vulnerable population followed by rapid dissemination $[16,31,35,36]$. The majority $(52 \%)$ of CRF01_AE infections in Bulgaria are PWID compared to only $4.8 \%$ in MSM, and PWID had at least six times greater odds of CRF01-AE infection than risk groups with other subtypes. In contrast, most persons with HIV-1 subtype B infections in Bulgaria are MSM [30].

We also observed that the proportion and odds of CRF01_AE infection in women was twice as high as those infected with other HIV-1 subtypes and a significantly higher percentage of these women were PWIDs (CRF01_AE 29/83 = 34.5\% vs. other subtypes $14 / 218=6.4 \% ; p<0.0001)$. These results correlate with the proportion of MSM being about 10 times as high in persons with non-CRF01_AE infections. Altogether, our studies show the independent introduction and spread of specific HIV-1 genotypes in different risk groups of the Bulgarian population $[13,30,31]$. For example, substance abuse can lead to risky sex and the further spread of HIV-1 and other sexually transmitted diseases, as we show in the current study for CRF01_AE $[16,30,32,36]$.

Since HIV-1 has a relatively high mutation rate, different genetic distance thresholds can be used to characterize the transmission network and inform the timing of probable infection and determine whether persons are infected with genetically similar or distant viruses [37]. Network analysis was performed at different genetic distance thresholds to analyze the transmission characteristics of CRF01_AE in Bulgaria. At the 1.5\% genetic distance, six clusters were identified, including one large outbreak cluster containing 154 members of which almost $41 \%$ consisted of young male PWID. These results confirm the spread of CRF01_AE infections within PWID and PWID/MSM, to a lesser extent in HET and at least MSM between 1995 and 2019. The small number of clusters $(n=3)$ with $\geq 3$ sequences and the dominance of one large cluster with most participants in the study supports the development of turbulent epidemic processes which have led to an outbreak involving mostly young, male PWID. The second and third largest clusters were also predominantly PWID and men. Only $17.0 \%$ of PWIDs were singletons or dyads, and only one in nine individuals from the most vulnerable MSM/PWID population was outside clusters. In contrast, singletons $(n=98)$ that did not cluster at this threshold were mostly HET and more evenly distributed between the sexes, indicating likely dead-end transmission events because the person sharing the related virus died, was not identified, or for whom an HIV-1 pol sequence was not obtained. Another possibility is that the infection was acquired abroad, which has been reported by $6.1 \%$ of singletons with these infections reportedly acquired in the UK, Turkey, Greece, Thailand, and Spain. However, the presence of CRF01_AE among different transmission risk groups, including MSM/PWID, 
HET, women, and newborns inside and outside of the clusters is indicative of possible transmission of HIV-1 CRF01_AE between risk groups and likely the general population.

In order to identify and characterize more recent transmissions in the network analysis, we reduced the genetic distance threshold to $0.5 \%$, which resulted in the formation of 10 clusters and reduced the number of participants in the large outbreak cluster to eight smaller clusters consisting of $18,12,7$, and two $(n=5)$ members. Similarly, the smaller clusters found at the $1.5 \%$ genetic distance disassociated into even smaller clusters or completely into singletons at $0.5 \%$. The clusters identified at the $0.5 \%$ cutoff contained mostly PWID and consisted of persons diagnosed from 2009 to 2019, which coincides with the period of outbreak growth in this population in Sofia (Figure 1 and Table 2). At both $d$ $=0.5 \%$ and $1.5 \%$, pol sequences from PWID were more likely to participate in transmission clusters, while sequences from HET and MSM were found less frequently in clusters. In general, these results suggest that although HIV-1 CRF01_AE was likely introduced into Bulgaria through HET transmission from infected persons from different countries, it then spread to PWID and continued to sustain this local sub-epidemic in Bulgaria.

Notably, we had to increase the genetic distance threshold to $3.5 \%$ to capture all taxa identified in the outbreak cluster by phylogenetic analysis. These different results likely reflect the long evolutionary history of CRF01_AE in Bulgaria over decades for which a larger genetic distance is required to detect those additional taxa in the transmission network. The $1.5 \%$ threshold used by most groups to identify relatively recent HIV-1 clusters was based on the genetic diversity of subtype B within a person over a period of about 8 years [38]. This distance threshold was later reduced to $0.5 \%$ to identify even more recent and rapidly growing clusters of concern $[33,38,39]$. Our cluster analysis results also suggest that non-B subtypes may require different genetic distant cutoffs to better identify transmission clusters, which is in line with other reports [40].

We found high assortativity by region further supporting a transmission network with more local genetic connections, which is similar to what we observed for the subtype B sub-epidemic in Bulgaria [30]. The signal of strong disassortativity by sex suggests that smaller clusters and strict thresholds seem to enrich for closely related, heterosexual pairs in Bulgaria. A mix of high assortativity $(r>>0)$ and high disassortativity $(r<<0)$ was observed with respect to transmission category, especially between dyads at strict and relaxed thresholds. It is important to note that assortativity comparisons between thresholds are necessarily comparing different clusters/dyads that contain different members. For example, dyads under a strict threshold will join to form larger clusters when a relaxed threshold is applied, and this principle is repeated for clusters of any scale.

In order to reconstruct the potential origin of CRF01_AE in Bulgaria, we conducted a global phylogenetic analysis of HIV-1 CRF01_AE sequences. Our overall CRF01_AE phylogenies, MRCA divergence dates, and evolutionary rate results from the Nextstrain analysis are consistent with those in the literature, including studies using complete genomes, providing strong support for our findings [8,41-43]. Phylogenetic analysis showed that after leaving Africa, CRF01_AE spread to and grew rapidly in Southeast Asia, including Thailand and Vietnam and then to China [5,8,44]. From Southeast Asia CRF01_AE spread to other countries, including Bulgaria. Our analysis inferred two different Bulgarian phylogenetic groups, including a large outbreak clade of 248 Bulgarian pol sequences mostly from PWID. Near the root of the outbreak clade were sequences from Vietnam and China with the Nextstrain analysis inferring Vietnam as the potential origin of the other sequences in the outbreak clade. Our analyses support our previous findings using smaller numbers of both Bulgarian and Asian sequences [13] and likely reflect the international employment agreement between Bulgaria and Vietnam in 1980 and 1990 for Vietnamese workers to come to Bulgaria and which overlaps with the inferred MRCA between 1988 and 1990 for the outbreak clade. These Vietnamese immigrants lived mostly in the capital city of Sofia where the majority $(60.7 \%)$ of the CRF01_AE cases were diagnosed. A total of about 35,000 Vietnamese immigrants were estimated to be in Bulgaria from 1980 to 1991, but most returned to Vietnam in 1991 after Bulgaria became an independent 
democracy in 1991. Combined, these results and historical information suggest a possible introduction of CRF01_AE into Bulgaria from Vietnam immigrants between 1988 and 1990 with subsequent rapid transmission via PWIDs, which is similar to other recent global outbreaks [35,36,45-48].

The outbreak clade also includes two sequences each from the Czech Republic and the UK. These findings are consistent results of a previous study on the global distribution of CRF01_AE that identified potential exports of CRF01_AE from Bulgaria to the Czech Republic [44], but we now report the dissemination of CRF01_AE from Bulgaria to the UK, China, and back to Vietnam from this outbreak cluster. A recent study of the intercontinental spread of CRF01_AE also found exportation to the UK via heterosexual transmission but from Thailand and Vietnam [44,49]. These findings could also be due to infections acquired while visiting Bulgaria, since frequent tourism occurs in Bulgaria, which is known for its beaches and mountains. Molecular dating inferred that the MRCA for the outbreak clade was late 1988, which precedes the first diagnosis of someone with this HIV-1 strain in Bulgaria by about 7 years, suggesting infections that may have been missed.

The smaller 19-person clade was located separately on the phylogenetic tree, and the MRCA for the clade was estimated to have on occurred 3/11/89 (CI 6/4/88-6/10/90), indicating early introduction into Bulgaria. These results likely reflect the risk practices of the participants at the beginning of the CRF01_AE sub-epidemic in Bulgaria from 1995 to 2007 in which the majority (75\%) of the first 56 cases were HET, followed by PWID (23.2\%), MTC (5.4\%), and MSM (1.8\%) [13]. These findings also suggest the possibility of multiple introductions of CRF01_AE into Bulgaria from Asia.

The CRF01_AE outbreak in PWIDS resulted in major public health responses by the Bulgarian Ministry of Health and NGOs, including HIV prevention and education campaigns, including a range of harm reduction services such as syringe exchange programs, condom distribution, HIV and Hepatitis testing, and other outreach activities. Opioid substitution therapy (OST) with methadone has been in place in Bulgaria since 1995. While these measures have significantly reduced HIV diagnoses in PWID, overall, the recent trend of increased infections in PWID may be explained by multiple factors. For example, funding from the Global Fund to Fight AIDS, Tuberculosis, and Malaria to combating HIV and TB in Bulgaria expired in 2015 reducing support for prevention services. The availability of OST providers in Bulgaria for PWID is limited, making it difficult to sustain. Additional public health strategies that address these limitations are required to prevent the spread of HIV-1 in PWIDs in Bulgaria. However, despite significant efforts by public health programs and NGOs to provide educational and prevention campaigns, HIV continues to be a significant burden for PWID in Bulgaria and other European countries [29,50].

Our study also has some potential limitations. Although this is the most comprehensive study of all HIV-1 subtype CRF01_AE sequences from Bulgaria through 2019, our study population included only persons for whom the HIV-1 pol gene was successfully obtained. As with all molecular epidemiologic studies, the inability to sequence HIV-1 from all infected persons, including from persons on treatment and with low viral loads or from persons not yet diagnosed, may affect our analyses and conclusions. Our results are also limited to the pol region of HIV-1. Using complete genomes may provide more accurate inference of transmission histories, especially in regions where multiple subtypes persist as in Bulgaria [43]. During the early years of the epidemic, resistance tests were not performed on all patients, some of whom may have gone abroad or died without a sample remaining for further testing, which may limit conclusions on transmission histories during this period. Some individuals in our study reported acquiring infection abroad, which may be influenced by recall bias for the potential place of infection. Samples from PWID were collected following a centralized system for registration and diagnosis of individuals with HIV in Bulgaria. Nonetheless, we cannot exclude that our results are fully representative of all HIV-infected PWID in Bulgaria. 


\section{Conclusions}

We applied a detailed phylodynamics approach to better understand the molecular epidemiology of HIV-1 subtype CRF01_AE infections in Bulgaria. Our findings indicate that CRF01_AE was likely introduced into Bulgaria from Asian countries multiple times since 1995 with infections rapidly spread among PWID and their contacts. These risk behaviors continue to spread CRF01_AE subtype infection in Bulgaria. Additional funding for prevention strategies in PWID are needed and should continue to include increased testing and treatment, and pre-exposure prophylaxis.

Author Contributions: W.M.S. and I.A. conceived and designed the study; I.A. assembled the data; W.M.S., I.A., E.M.C., Y.P., and S.K. analyzed the data; I.A. and W.M.S. prepared the draft manuscript. L.G., R.D., A.P., A.G., A.K., C.S.-D., I.E. and N.Y. reviewed the draft and all authors contributed to the final version, which was approved by all authors for submission. All authors have read and agreed to the published version of the manuscript.

Funding: This work was supported in part by a grant from the Ministry of Education and Science, Bulgaria (contract: DN03/2-16.12.2016); by the National Center of Infectious and Parasitic Diseases, Sofia, Bulgaria; by the National Program for the Prevention and Control of HIV and Sexually Transmitted Infections, Bulgaria; by the European Regional Development Fund through Operational Program Science and Education for Smart Growth 2014-2020, Grant BG05M2OP001-1.002-0001-C04 "Fundamental Translational and Clinical Investigations on Infections and Immunity".

Institutional Review Board Statement: This study was approved by the Ethical Committee at the National Centre of Infectious and Parasitic Diseases, Sofia, Bulgaria (NCIPD IRB 00006384). A nonresearch determination (protocol \#6832) was approved at CDC for analysis of the HIV sequences in this study.

Informed Consent Statement: Informed consent was obtained from all subjects involved in the study.

Data Availability Statement: The sequences analyzed for this study under accession names described in the text are openly available at GenBank (https:/ /www.ncbi.nlm.nih.gov/genbank/).

Acknowledgments: Use of trade names is for identification only and does not imply endorsement by the U.S. Department of Health and Human Services, the Public Health Service, or the Centers for Disease Control and Prevention (CDC). The findings and conclusions in this report are those of the authors and do not necessarily represent the views of the CDC, or any of the authors' affiliated institutions.

Conflicts of Interest: The authors declare no conflict of interest. The funding sponsors had no role in the design of the study; in the collection, analyses, or interpretation of data; in the writing of the manuscript, and in the decision to publish the results.

\section{References}

1. Leitner, T. The Puzzle of HIV Neutral and Selective Evolution. Mol. Biol. Evol. 2018, 35, 1355-1358. [CrossRef] [PubMed]

2. Coffin, J.M. HIV population dynamics in vivo: Implications for genetic variation, pathogenesis, and therapy. Science 1995, 267, 483-489. [CrossRef] [PubMed]

3. Luo, G.X.; Taylor, J. Template switching by reverse transcriptase during DNA synthesis. J. Virol. 1990, 64, 4321-4328. [CrossRef]

4. Sharp, P.M.; Hahn, B.H. Origins of HIV and the AIDS Pandemic. Cold Spring Harb. Perspect. Med. 2011, 1, a006841. [CrossRef] [PubMed]

5. Hemelaar, J.; Elangovan, R.; Yun, J.; Dickson-Tetteh, L.; Fleminger, I.; Kirtley, S.; Williams, B.; Gouws-Williams, E.; Ghys, P.D.; Abimiku, A.G.; et al. Global and regional molecular epidemiology of HIV-1, 1990-2015: A systematic review, global survey, and trend analysis. Lancet Infect. Dis. 2019, 19, 143-155. [CrossRef]

6. Carr, J.K.; O Salminen, M.; Koch, C.; Gotte, D.; Artenstein, A.W.; A Hegerich, P.; Louis, D.S.; Burke, D.S.; E McCutchan, F. Full-length sequence and mosaic structure of a human immunodeficiency virus type 1 isolate from Thailand. J. Virol. 1996, 70, 5935-5943. [CrossRef]

7. McCutchan, F.E. Global epidemiology of HIV. J. Med Virol. 2006, 78, S7-S12. [CrossRef]

8. Junqueira, D.M.; Wilkinson, E.; Vallari, A.S.; Deng, X.; Achari, A.; Yu, G.; McArthur, C.P.; Kaptue, L.; Mbanya, D.N.; Chiu, C.Y.; et al. New Genomes from the Congo Basin Expand History of CRF01_AE Origin and Dissemination. AIDS Res. Hum. Retrovir. 2020, 36, 574-582. [CrossRef] 
9. Hofstra, L.M.; Sauvageot, N.; Albert, J.; Alexiev, I.; Garcia, F.P.; Struck, D.; Van De Vijver, D.A.M.C.; Åsjö, B.; Beshkov, D.; Coughlan, S.; et al. Transmission of HIV Drug Resistance and the Predicted Effect on Current First-line Regimens in Europe. Clin. Infect. Dis. 2016, 62, 655-663. [CrossRef]

10. Stanojevic, M.; Alexiev, I.; Beshkov, D.; Gokengin, D.; Mezei, M.; Minarovits, J.; Otelea, D.; Paraschiv, S.; Poljak, M.; Zidovec, S.L.; et al. HIV1 Molecular Epidemiology in the Balkans-A Melting Pot for High Genetic Diversity. Aids. Rev. 2012, $14,28-36$. [PubMed]

11. Alexiev, I.; Beshkov, D.; Shankar, A.; Hanson, D.L.; Paraskevis, D.; Georgieva, V.; Karamacheva, L.; Taskov, H.; Varleva, T.; Elenkov, I.; et al. Detailed Molecular Epidemiologic Characterization of HIV-1 Infection in Bulgaria Reveals Broad Diversity and Evolving Phylodynamics. PLoS ONE 2013, 8, e59666. [CrossRef]

12. Alexiev, I.; Shankar, A.; Wensing, A.M.J.; Beshkov, D.; Elenkov, I.; Stoycheva, M.; Nikolova, D.; Nikolova, M.; Switzer, W.M. Low HIV-1 transmitted drug resistance in Bulgaria against a background of high clade diversity. J. Antimicrob. Chemother. 2015, 70, 1874-1880. [CrossRef] [PubMed]

13. Alexiev, I.; Shankar, A.; Dimitrova, R.; Gancheva, A.; Kostadinova, A.; Teoharov, P.; Golkocheva, E.; Nikolova, M.; Muhtarova, M.; Elenkov, I.; et al. Origin and spread of HIV-1 in persons who inject drugs in Bulgaria. Infect. Genet. Evol. 2016, 46, 269-278. [CrossRef] [PubMed]

14. Struck, D.; Lawyer, G.; Ternes, A.-M.; Schmit, J.-C.; Bercoff, D.P. COMET: Adaptive context-based modeling for ultrafast HIV-1 subtype identification. Nucleic Acids Res. 2014, 42, e144. [CrossRef] [PubMed]

15. Pineda-Pena, A.; Faria, N.R.; Imbrechts, S.; Libin, P.; Abecasis, A.B.; Deforche, K.; Gómez-López, A.; Camacho, R.J.; De Oliveira, T.; Vandamme, A.-M. Automated subtyping of HIV-1 genetic sequences for clinical and surveillance purposes: Performance evaluation of the new REGA version 3 and seven other tools. Infect. Genet. Evol. 2013, 19, 337-348. [CrossRef]

16. Schultz, A.-K.; Zhang, M.; Bulla, I.; Leitner, T.; Korber, B.; Morgenstern, B.; Stanke, M. jpHMM: Improving the reliability of recombination prediction in HIV-1. Nucleic Acids Res. 2009, 37, W647-W651. [CrossRef]

17. Lole, K.S.; Bollinger, R.C.; Paranjape, R.S.; Gadkari, D.; Kulkarni, S.S.; Novak, N.G.; Ingersoll, R.; Sheppard, H.W.; Ray, S.C. Full-Length Human Immunodeficiency Virus Type 1 Genomes from Subtype C-Infected Seroconverters in India, with Evidence of Intersubtype Recombination. J. Virol. 1999, 73, 152-160. [CrossRef]

18. Larsson, A. AliView: A fast and lightweight alignment viewer and editor for large datasets. Bioinformatics 2014, 30, 3276-3278. [CrossRef]

19. Edgar, R.C. MUSCLE: Multiple sequence alignment with high accuracy and high throughput. Nucleic Acids Res. 2004, 32, 1792-1797. [CrossRef]

20. Katoh, K.; Rozewicki, J.; Yamada, K.D. MAFFT online service: Multiple sequence alignment, interactive sequence choice and visualization. Brief. Bioinform. 2019, 20, 1160-1166. [CrossRef]

21. Kuraku, S.; Zmasek, C.M.; Nishimura, O.; Katoh, K. aLeaves facilitates on-demand exploration of metazoan gene family trees on MAFFT sequence alignment server with enhanced interactivity. Nucleic Acids Res. 2013, 41, W22-W28. [CrossRef] [PubMed]

22. Price, M.N.; Dehal, P.S.; Arkin, A.P. FastTree 2-Approximately Maximum-Likelihood Trees for Large Alignments. PLoS ONE 2010, 5, e9490. [CrossRef] [PubMed]

23. Hadfield, J.; Megill, C.; Bell, S.M.; Huddleston, J.; Potter, B.; Callender, C.; Sagulenko, P.; Bedford, T.; A Neher, R. Nextstrain: Real-time tracking of pathogen evolution. Bioinformatics 2018, 34, 4121-4123. [CrossRef] [PubMed]

24. Sagulenko, P.; Puller, V.; Neher, R.A. TreeTime: Maximum-likelihood phylodynamic analysis. Virus Evol. 2018, 4, vex042. [CrossRef] [PubMed]

25. Campbell, E.; Boylesm, A.; Shankar, A.; Kim, J.; Knyazev, S.; Switzer, W.M. MicrobeTrace: Retooling Molecular Epidemiology for Rapid Public Health Response. bioRxiv 2020. [CrossRef]

26. Attribute Assortativity. Available online: https://github.com/Sergey-Knyazev/attribute_assortativity (accessed on 28 October 2020).

27. NetworkX. Available online: https:/ / networkx.github.io (accessed on 28 October 2020).

28. Hai-Long, H.; Jian, Z.; Ping-Ping, Y.; Liang, C.; Xun, L.; Shan, Z.Z.; Yan-Sheng, Y. Genetic Characterization of CRF01_AE Full-Length Human Immunodeficiency Virus Type 1 Sequences from Fujian, China. AIDS Res. Hum. Retrovir. 2007, 23, 569-574. [CrossRef]

29. Billings, E.A.; Heipertz, R.A.; Varleva, T.; Sanders-Buell, E.; O’Sullivan, A.M.; Bose, M.; Howell, S.; Kijak, G.H.; Taskov, H.; Elenkov, I.; et al. HIV-1 genetic diversity and demographic characteristics in Bulgaria. PLoS ONE 2019, 14, e0217063. [CrossRef]

30. Alexiev, I.; Campbell, E.; Knyazev, S.; Pan, Y.; Grigorova, L.; Dimitrova, R.; Partsuneva, A.; Gancheva, A.; Kostadinova, A.; Seguin-Devaux, C.; et al. Molecular Epidemiology of the HIV-1 Subtype B Sub-Epidemic in Bulgaria. Viruses 2020, $12,441$. [CrossRef]

31. Campbell, E.M.; Jia, H.; Shankar, A.; Hanson, D.; Luo, W.; Masciotra, S.; Owen, S.M.; Oster, A.M.; Galang, R.R.; Spiller, M.W.; et al. Detailed Transmission Network Analysis of a Large Opiate-Driven Outbreak of HIV Infection in the United States. J. Infect. Dis. 2017, 216, 1053-1062. [CrossRef]

32. Hassan, A.S.; Pybus, O.G.; Sanders, E.J.; Albert, J.; Esbjörnsson, J. Defining HIV-1 transmission clusters based on sequence data. AIDS 2017, 31, 1211-1222. [CrossRef] 
33. Oster, A.M.; France, A.M.; Panneer, N.; Ocfemia, M.C.B.; Campbell, E.; Dasgupta, S.; Switzer, W.M.; Wertheim, J.O.; Hernandez, A.L. Identifying Clusters of Recent and Rapid HIV Transmission Through Analysis of Molecular Surveillance Data. JAIDS J. Acquir. Immune Defic. Syndr. 2018, 79, 543-550. [CrossRef]

34. Rich, S.; Richards, V.L.; Mavian, C.; Switzer, W.M.; Magalis, B.R.; Poschman, K.; Geary, S.; Broadway, S.E.; Bennett, S.B.; Blanton, J.; et al. Employing Molecular Phylodynamic Methods to Identify and Forecast HIV Transmission Clusters in Public Health Settings: A Qualitative Study. Viruses 2020, 12, 921. [CrossRef]

35. Paraskevis, D.; Nikolopoulos, G.; Tsiara, C.; Paraskeva, D.; Antoniadou, A.; Lazanas, M.; Gargalianos, P.; Psychogiou, M.; Malliori, M.; Kremastinou, J.; et al. HIV-1 outbreak among injecting drug users in Greece, 2011: A preliminary report. Eurosurveillance 2011, 16, 19962. [CrossRef]

36. Arendt, V.; Guillorit, L.; Origer, A.; Sauvageot, N.; Vaillant, M.; Fischer, A.; Goedertz, H.; François, J.-H.; Alexiev, I.; Staub, T.; et al. Injection of cocaine is associated with a recent HIV outbreak in people who inject drugs in Luxembourg. PLoS ONE 2019, 14, e0215570. [CrossRef] [PubMed]

37. Hightower, G.K.; May, S.J.; Pérez-Santiago, J.; Pacold, M.E.; Wagner, G.A.; Little, S.J.; Richman, D.D.; Mehta, S.R.; Smith, D.M.; Pond, S.L.K. HIV-1 Clade B pol Evolution following Primary Infection. PLoS ONE 2013, 8, e68188. [CrossRef] [PubMed]

38. Centers for Disease Control and Prevention. Detecting and Responding to HIV Transmission Clusters. A Guide for Health Departments. June 2018. Available online: https:/ / www.cdc.gov/hiv/pdf/funding/announcements/ps18-1802/CDC-HIV-PS1 8-1802-AttachmentE-Detecting-Investigating-and-Responding-to-HIV-Transmission-Clusters.pdf (accessed on 28 October 2020).

39. Campbell, E.; Patala, A.; Shankar, A.; Li, J.-F.; Johnson, J.A.; Westheimer, E.; Gay, C.L.; Cohen, S.E.; Switzer, W.M.; Peters, P.J. Phylodynamic Analysis Complements Partner Services by Identifying Acute and Unreported HIV Transmission. Viruses 2020, 12, 145. [CrossRef] [PubMed]

40. Novitsky, V.; Steingrimsson, J.A.; Howison, M.; Gillani, F.S.; Li, Y.; Manne, A.; Fulton, J.; Spence, M.; Parillo, Z.; Marak, T.; et al. Empirical comparison of analytical approaches for identifying molecular HIV-1 clusters. Sci. Rep. 2020, 10, 1-11. [CrossRef] [PubMed]

41. Feng, Y.; He, X.; Hsi, J.H.; Li, F.; Li, X.; Wang, Q.; Ruan, Y.; Xing, H.; Lam, T.T.-Y.; Pybus, O.G.; et al. The rapidly expanding CRF01_AE epidemic in China is driven by multiple lineages of HIV-1 viruses introduced in the 1990s. AIDS 2013, 27, 1793-1802. [CrossRef]

42. Li, X.; Xue, Y.; Lin, Y.; Gai, J.; Zhang, L.; Cheng, H.; Ning, Z.; Zhou, L.; Zhu, K.; Vanham, G.; et al. Evolutionary Dynamics and Complicated Genetic Transmission Network Patterns of HIV-1 CRF01_AE among MSM in Shanghai, China. Sci. Rep. 2016, 6, 34729. [CrossRef]

43. Li, X.; Liu, H.; Liu, L.; Feng, Y.; Kalish, M.L.; Ho, S.Y.W.; Shao, Y. Tracing the epidemic history of HIV-1 CRF01_AE clusters using near-complete genome sequences. Sci. Rep. 2017, 7, 1-11. [CrossRef]

44. Angelis, K.; Albert, J.; Mamais, I.; Magiorkinis, G.; Hatzakis, A.; Hamouda, O.; Struck, D.; Vercauteren, J.; Wensing, A.M.J.; Alexiev, I.; et al. Global Dispersal Pattern of HIV Type 1 Subtype CRF01_AE: A Genetic Trace of Human Mobility Related to Heterosexual Sexual Activities Centralized in Southeast Asia. J. Infect. Dis. 2014, 211, 1735-1744. [CrossRef] [PubMed]

45. Palumbo, P.J.; Zhang, Y.; Fogel, J.M.; Guo, X.; Clarke, W.; Breaud, A.; Richardson, P.; Piwowar-Manning, E.; Hart, S.; Hamilton, E.L.; et al. HIV drug resistance in persons who inject drugs enrolled in an HIV prevention trial in Indonesia, Ukraine, and Vietnam: HPTN 074. PLoS ONE 2019, 14, e0223829. [CrossRef] [PubMed]

46. Lai, A.; Bozzi, G.; Franzetti, M.; Binda, F.; Simonetti, F.R.; De Luca, A.; Micheli, V.; Meraviglia, P.; Bagnarelli, P.; Di Biagio, A.; et al. HIV-1 A1 Subtype Epidemic in Italy Originated from Africa and Eastern Europe and Shows a High Frequency of Transmission Chains Involving Intravenous Drug Users. PLoS ONE 2016, 11, e0146097. [CrossRef] [PubMed]

47. Paraschiv, S.; Bănică, L.; Nicolae, I.; Niculescu, I.; Abagiu, A.; Jipa, R.; Pineda-Pena, A.; Pingarilho, M.; Neaga, E.; Theys, K.; et al. Epidemic dispersion of HIV and HCV in a population of co-infected Romanian injecting drug users. PLoS ONE 2017, 12, e0185866. [CrossRef] [PubMed]

48. Golden, M.R.; Lechtenberg, R.; Glick, S.N.; Dombrowski, J.; Duchin, J.; Reuer, J.R.; Dhanireddy, S.; Neme, S.; Buskin, S.E. Outbreak of Human Immunodeficiency Virus Infection Among Heterosexual Persons Who Are Living Homeless and Inject Drugs-Seattle, Washington, 2018. MMWR. Morb. Mortal. Wkly. Rep. 2019, 68, 344-349. [CrossRef]

49. An, M.; Han, X.; Zhao, B.; English, S.; Frost, S.D.W.; Zhang, H.; Shang, H. Cross-Continental Dispersal of Major HIV-1 CRF01_AE Clusters in China. Front. Microbiol. 2020, 11, 61. [CrossRef]

50. World Health Organization. HIV/AIDS Surveillance in Europe: 2019. Available online: https:/ /www.ecdc.europa.eu/sites/ default/files / documents / HIV-annual-surveillance-report-2019.pdf (accessed on 28 October 2020). 